Article

\title{
Behavior of Non-Oscillatory Solutions of Fourth-Order Neutral Differential Equations
}

\author{
Osama Moaaz $^{1}$ (D), Rami Ahmad El-Nabulsi ${ }^{2, *}$ and Omar Bazighifan ${ }^{3,4}$ (D) \\ 1 Department of Mathematics, Faculty of Science, Mansoura University, 35516 Mansoura, Egypt; \\ o_moaaz@mans.edu.eg \\ 2 Athens Institute for Education and Research, Mathematics and Physics Divisions, 10671 Athens, Greece \\ 3 Department of Mathematics, Faculty of Science, Hadhramout University, 50512 Hadhramout, Yemen; \\ o.bazighifan@gmail.com \\ 4 Department of Mathematics, Faculty of of Education, Seiyun University, 50512 Hadhramout, Yemen \\ * Correspondence: nabulsiahmadrami@yahoo.fr
}

Received: 12 February 2020; Accepted: 7 March 2020; Published: 19 March 2020

\begin{abstract}
In this paper, we deal with the asymptotics and oscillation of the solutions of fourth-order neutral differential equations of the form $\left(r(t)\left(z^{\prime \prime \prime}(t)\right)^{\alpha}\right)^{\prime}+q(t) x^{\alpha}(g(t))=0$, where $z(t):=$ $x(t)+p(t) x(\delta(t))$. By using a generalized Riccati transformation, we study asymptotic behavior and derive some new oscillation criteria. Our results extend and improve some well-known results which were published recently in the literature. Symmetry ideas are often invisible in these studies, but they help us decide the right way to study them, and to show us the correct direction for future developments. An example is given to illustrate the importance of our results.
\end{abstract}

Keywords: fourth-order differential equations; neutral delay; oscillation

\section{Introduction}

To date, the oscillatory behavior of the solutions to differential equations has been discussed in many papers. Among them, there are many papers about the oscillation of the solutions to functional differential equations. In a related field, the asymptotic behavior of the solutions to delay and neutral delay differential equations were discussed in many works, and there have been very fruitful achievements see [1-28].

In this paper, our focus is on improving the criteria of oscillation of fourth-order neutral equations

$$
\left(r(t)\left(z^{\prime \prime \prime}(t)\right)^{\alpha}\right)^{\prime}+q(t) x^{\alpha}(g(t))=0
$$

where $t \geq t_{0}$ and $z(t):=x(t)+p(t) x(\delta(t))$. In this work, we assume:

Hypothesis 1. $\alpha \in\left\{a / b: a, b \in \mathbb{Z}^{+}\right\}, r \in C^{1}\left(\left[t_{0}, \infty\right)\right), r(t)>0, r^{\prime}(t) \geq 0$ and

$$
\theta\left(t_{0}\right):=\int_{t_{0}}^{\infty} r^{-1 / \alpha}(s) \mathrm{d} s<\infty
$$

Hypothesis 2. $p, q \in C\left(\left[t_{0}, \infty\right)\right), q(t)>0,0 \leq p(t)<p_{0}<\infty$,

Hypothesis 3. $\delta \in C^{1}\left(\left[t_{0}, \infty\right)\right), g \in C\left(\left[t_{0}, \infty\right)\right), \delta^{\prime}(t)>0, \delta(t) \leq t$ and $\lim _{t \rightarrow \infty} \delta(t)=\lim _{t \rightarrow \infty} g(t)=\infty$.

By a solution of (1) we mean a function $x \in C^{3}\left(\left[t_{x}, \infty\right)\right), t_{x} \geq t_{0}$, which has the property $\left(r(t)\left(z^{\prime \prime \prime}(t)\right)^{\alpha}\right) \in C^{1}\left(\left[t_{x}, \infty\right)\right)$, and satisfies (1) on $\left[t_{x}, \infty\right)$. We consider only those solutions $x$ of (1) 
which satisfy $\sup \{|x(t)|: t \geq T\}>0$, for all $T \geq t_{x}$. A solution $x$ of (1) is said to be non-oscillatory if it is positive or negative, ultimately; otherwise, it is said to be oscillatory.

Delay differential equations are often studied in one of two cases

$$
\int_{t_{0}}^{\infty} r^{-1 / \alpha}(s) \mathrm{d} s=\infty
$$

or (2) which it is said to be in canonical or noncanonical. For canonical, Moaaz et al. [21] proved that (1) is oscillatory if

$$
\liminf _{t \rightarrow \infty} \int_{\delta^{-1}(\eta(t))}^{t}\left(\frac{\left(\delta^{-1}(\eta(s))\right)^{n-1}}{r^{1 / \alpha}\left(\delta^{-1}(\eta(s))\right)}\right)^{\alpha} q(s) P_{n}^{\alpha}(g(s)) \mathrm{d} s>\frac{((n-1) !)^{\alpha}}{\mathrm{e}}
$$

and

$$
\liminf _{t \rightarrow \infty} \int_{\delta^{-1}(\zeta(t))}^{t} \delta^{-1}(\zeta(s)) R_{n-3}(s) \mathrm{d} s>\frac{1}{\mathrm{e}^{\prime}}
$$

where $P_{n}(t)=1 / p\left(\delta^{-1}(t)\right)\left(1-\left(\left(\delta^{-1}\left(\delta^{-1}(t)\right)\right)^{n-1} /\left(\delta^{-1}(t)\right)^{k-1} p\left(\delta^{-1}\left(\delta^{-1}(t)\right)\right)\right)\right)$.

In [25], the authors proved that (1) is oscillatory if the first-order differential equation

$$
y^{\prime}(t)+q(t)(1-p(\delta(t)))^{\alpha}\left(\frac{\lambda \delta^{n-1}(t)}{(n-1) ! r^{1 / \alpha}(\delta(t))}\right)^{\alpha} y(\delta(t))=0,
$$

is oscillatory, also

$$
\limsup _{t \rightarrow \infty} \int_{t_{0}}^{t}\left(q(s)(1-p(\delta(s)))^{\alpha}\left(\frac{\lambda_{1} \delta^{n-2}(s) \delta(s)}{(n-2) !}\right)^{\alpha}-\frac{\alpha^{\alpha+1}}{(\alpha+1)^{\alpha+1} \delta(s) r^{1 / \alpha}(s)}\right) \mathrm{d} s=\infty
$$

and

$$
\limsup _{t \rightarrow \infty} \int_{t_{0}}^{t}\left(q(s)\left(\frac{\delta^{n-2}(s) \delta(s)}{(n-2) !}\right)^{\alpha}-\frac{\alpha^{\alpha+1}}{(\alpha+1)^{\alpha+1} \delta(s) r^{1 / \alpha}(s)}\right) \mathrm{d} s=\infty .
$$

Now, we state some lemmas that will be useful in establishing our main results:

Lemma 1 ([18]). If the function $x$ satisfies $x^{(i)}(t)>0, i=0,1, \ldots, n$, and $x^{(n+1)}(t)<0$, then

$$
\frac{x(t)}{t^{n} / n !} \geq \frac{x^{\prime}(t)}{t^{n-1} /(n-1) !} .
$$

Lemma 2 ([6] (Lemma 2.2.3)). Let $x \in C^{n}\left(\left[t_{0}, \infty\right),(0, \infty)\right)$. Assume that $x^{(n)}(t)$ is of fixed sign and not identically zero on $\left[t_{0}, \infty\right)$ and that there exists $a t_{1} \geq t_{0}$ such that $x^{(n-1)}(t) x^{(n)}(t) \leq 0$ for all $t \geq t_{1}$. If $\lim _{t \rightarrow \infty} x(t) \neq 0$, then for every $\mu \in(0,1)$ there exists $t_{\mu} \geq t_{1}$ such that

$$
x(t) \geq \frac{\mu}{(n-1) !} t^{n-1}\left|x^{(n-1)}(t)\right| \text { for } t \geq t_{\mu} .
$$

Lemma 3 ([10]). Let $\beta$ be a ratio of two odd numbers, $C>0$ and $D$ are constants. Then

$$
D x-C x^{(\beta+1) / \beta} \leq \frac{\beta^{\beta}}{(\beta+1)^{\beta+1}} \frac{D^{\beta+1}}{C^{\beta}} .
$$

In this work, we obtain some new oscillation criteria for (1). The paper is organized as follows. Firstly, we study the behavior of non-oscillatory solutions of (1) andwe obtain the sufficient conditions which guarantee that every non-oscillatory solution of (1) tends to zero. Secondly, we will use the 
Riccati transformation technique to give some conditions for the oscillation of (1). Finally, an example is provided to illustrate the main results.

\section{The Behavior of Non-Oscillatory Solutions}

In this section, we study the behavior of non-oscillatory solutions of (1) when $p_{0} \in(0,1)$. We use an approach that leads to only three independent conditions, but we obtain sufficient conditions which guarantee that every non-oscillatory solution of (1) tends to zero.

Definition 1. A solution $x$ of (1) is said to be non-oscillatory if it is positive or negative; otherwise, it is said to be oscillatory.

Lemma 4. Assume that $x$ is an eventually positive solution of $(1)$. Then, $r(t)\left(z^{\prime \prime \prime}(t)\right)^{\alpha}$ is non-increasing. Moreover, we have the following cases:

$$
\begin{array}{ll}
\left(S_{1}\right): & z^{\prime}(t)>0, z^{\prime \prime}(t)>0, z^{\prime \prime \prime}(t)>0 \text { and } z^{(4)}(t)<0 ; \\
\left(S_{2}\right): & z^{\prime}(t)>0, z^{\prime \prime}(t)<0, z^{\prime \prime \prime}(t)>0 \text { and } z^{(4)}(t)<0 ; \\
\left(S_{3}\right): & z^{\prime}(t)>0, z^{\prime \prime}(t)>0 \text { and } z^{\prime \prime \prime}(t)<0 ; \\
\left(S_{4}\right): & z^{\prime}(t)<0, z^{\prime \prime}(t)>0 \text { and } z^{\prime \prime \prime}(t)<0 .
\end{array}
$$

Lemma 5. Let $x$ be a positive solution of (1) with property $\left(S_{1}\right)$ or $\left(S_{2}\right)$. Then the equation

$$
w^{\prime}(t)+\left(1-p_{0}\right)^{\alpha} \frac{q(t)}{r(g(t))}\left(\frac{\mu}{6} g^{3}(t)\right)^{\alpha} w(g(t))=0
$$

has a non-oscillatory solution.

Proof. Suppose the $x$ is a positive solution of (1) with property $\left(S_{1}\right)$ or $\left(S_{2}\right)$. Then, we have that

$$
z^{\prime}(t)>0, z^{\prime \prime \prime}(t)>0 \text { and } z^{(4)}(t)<0 .
$$

Thus, from Lemma 2, we obtain

$$
z(t) \geq \frac{\mu}{6} t^{3} z^{\prime \prime \prime}(t)
$$

From definition of $z$, we see that $x(t) \geq\left(1-p_{0}\right) z(t)$, which with (1) gives

$$
\left(r(t)\left(z^{\prime \prime \prime}(t)\right)^{\alpha}\right)^{\prime}+\left(1-p_{0}\right)^{\alpha} q(t) z^{\alpha}(g(t)) \leq 0 .
$$

Hence, from (7), if we set $w:=r\left(z^{\prime \prime \prime}\right)^{\alpha}>0$, then the differential inequality

$$
w^{\prime}(t)+\left(1-p_{0}\right)^{\alpha} \frac{q(t)}{r(g(t))}\left(\frac{\mu}{6} g^{3}(t)\right)^{\alpha} w(g(t)) \leq 0 .
$$

From [4] (Corollary 1), we have that (6) also has a positive solution, and this completes the proof.

Lemma 6. Let $x$ be a positive solution of (1) with property $\left(S_{3}\right)$. Then the equation

$$
\left(r(t)\left(\omega^{\prime}(t)\right)^{\alpha}\right)^{\prime}+\left(1-p_{0}\right)^{\alpha} q(t)\left(\frac{\mu}{2} g^{2}(t)\right)^{\alpha} \omega^{\alpha}(t)=0,
$$

has a non-oscillatory solution.

Proof. Suppose the $x$ is a positive solution of (1) with property $\left(S_{3}\right)$. Using Lemma 2, we obtain

$$
z(t) \geq \frac{\mu}{2} t^{2} z^{\prime \prime}(t)
$$


As in the proof of Lemma 6, we can obtain that (8). Next, if we set $G:=r\left(z^{\prime \prime \prime} / z^{\prime \prime}\right)^{\alpha}<0$, then we get

$$
G^{\prime}(t) \leq-\left(1-p_{0}\right)^{\alpha} q(t) \frac{z^{\alpha}(g(t))}{\left(z^{\prime \prime}(t)\right)^{\alpha}}-\alpha r^{-1 / \alpha}(t) G^{1+1 / \alpha}(t) .
$$

Hence, from the fact that $z^{\prime \prime \prime}<0$ and (10), we find

$$
G^{\prime}(t)+\left(1-p_{0}\right)^{\alpha} q(t)\left(\frac{\mu}{2} g^{2}(t)\right)^{\alpha}+\alpha r^{-1 / \alpha}(t) G^{1+1 / \alpha}(t) \leq 0 .
$$

Therefore, there exists a function $G \in C^{1}\left(\left[t_{0}, \infty\right), \mathbb{R}\right)$ such that (11) holds. It follow from [1] that (9) has a non-oscillatory solution, and this completes the proof.

Theorem 1. Assume that the differential equations (6) and (9) are oscillatory. Then every non-oscillatory solution of (1) tends to zero if

$$
\int_{t_{0}}^{\infty}\left(\frac{1}{r(u)} \int_{t_{0}}^{t} q(s) \mathrm{d} s\right)^{1 / \alpha} \mathrm{d} u=\infty
$$

Proof. Assume the contrary that $x$ is a positive solution of (1) with property $\lim _{t \rightarrow \infty} x(t) \neq 0$. From Lemma 4 , we have cases $\left(S_{1}\right)-\left(S_{4}\right)$. Using Lemmas 5 and 6 with the fact that the differential Equations (6) and (9) are oscillatory, we conclude that $x$ satisfies case $\left(S_{4}\right)$. Then, since $z$ is a positive decreasing function, we get that $\lim _{t \rightarrow \infty} z(t)=c \geq 0$. Suppose the contrary that $c>0$. Thus, for all $\varepsilon>0$ and $t$ enough large, we have $c \leq z(t)<c+\varepsilon$. Choosing $\varepsilon<\left(1-p_{0}\right)\left(c / p_{0}\right)$, we obtain

$$
\begin{aligned}
x(t) & =z(t)-p_{0}(t) x(\delta(t))>c-p_{0} z(\delta(t)) \\
& >L(\gamma+\varepsilon)>L z(t),
\end{aligned}
$$

where $L=\left(c-p_{0}(c+\varepsilon)\right) /(c+\varepsilon)>0$. Hence, from (1), we have

$$
\begin{aligned}
\left(r(t)\left(z^{\prime \prime \prime}(t)\right)^{\alpha}\right)^{\prime} & =-q(t) x^{\alpha}(g(t)) \leq-L^{\alpha} q(t) z^{\alpha}(g(t)) \\
& \leq-L^{\alpha} \varepsilon^{\alpha} q(t) .
\end{aligned}
$$

Integrating this inequality from $t_{1}$ to $t$, we get

$$
z^{\prime \prime \prime}(t) \leq-L \varepsilon\left(\frac{1}{r(t)} \int_{t_{1}}^{t} q(s) \mathrm{d} s\right)^{1 / \alpha} .
$$

By integrating from $t_{1}$ to $t$, we obtain

$$
z^{\prime \prime}(t) \leq z^{\prime \prime}\left(t_{1}\right)-L \varepsilon \int_{t_{1}}^{t}\left(\frac{1}{r(u)} \int_{t_{1}}^{t} q(s) \mathrm{d} s\right)^{1 / \alpha} \mathrm{d} u
$$

Letting $t \rightarrow \infty$ and taking into account (12), we get that $\lim _{t \rightarrow \infty} z^{\prime \prime}(t)=-\infty$. This contradicts the fact that $z^{\prime \prime}(t)>0$. Therefore, $c=0$; moreover the fact $x(t) \leq z(t)$ implies $\lim _{t \rightarrow \infty} x(t)=0$, a contradiction. This completes the proof.

Corollary 1. Assume that (12) holds. Then every non-oscillatory solution of (1) tends to zero if $\int_{t_{0}}^{\infty} q(s) \mathrm{d} s=\infty$,

$$
\liminf _{t \rightarrow \infty} \int_{g(t)}^{t} \frac{q(s) g^{3 \alpha}(s)}{r(g(s))} \mathrm{d} s>\frac{6^{\alpha}}{\mathrm{e} \mu^{\alpha}\left(1-p_{0}\right)^{\alpha}}
$$


and

$$
\limsup _{t \rightarrow \infty} \int_{t_{0}}^{t}\left(\left(1-p_{0}\right)^{\alpha} \theta^{\alpha}(s) q(s)\left(\frac{\mu}{2} g^{2}(s)\right)^{\alpha}-\left(\frac{\alpha}{\alpha+1}\right)^{\alpha+1} \frac{1}{r^{1 / \alpha}(s) \theta(s)}\right) \mathrm{d} s>0 .
$$

Proof. It is well-known from [3] (Theorem 2) and [2] (Corollary 2.8) that (14) and (15) imply oscillation of (6) and (9), respectively.

Lemma 7. Assume that $x$ is an eventually positive solution of (1). If $z$ is an increasing and

$$
p(t) \sum_{k=0}^{(n-1) / 2} \prod_{r=1}^{2 k} p\left(\delta^{i}(t)\right)<1
$$

then

$$
x(t) \geq(1-\widehat{p}(t)) z(t),
$$

for any odd positive integer $n$, where

$$
\widehat{p}(t):=p(t) \sum_{k=0}^{(n-1) / 2} \prod_{r=1}^{2 k} p\left(\delta^{i}(t)\right)
$$

Proof. From the definition of $z(t)$, we obtain

$$
\begin{aligned}
x(t) & =z(t)-p(t) x(\delta(t)) \\
& =z(t)-p(t) z(\delta(t))+p(t) p(\delta(t)) x\left(\delta^{2}(t)\right) \\
& =z(t)-p(t) z(\delta(t))-p(t) p(\delta(t)) p\left(\delta^{2}(t)\right) z\left(\delta^{3}(t)\right)+p(t) p(\delta(t)) p\left(\delta^{2}(t)\right) p\left(\delta^{3}(t)\right) x\left(\delta^{4}(t)\right) \\
& \geq z(t)-\sum_{k=0}^{(n-1) / 2} \prod_{r=0}^{2 k} p\left(\delta^{i}(t)\right) z\left(\delta^{2 k+1}(t)\right)+\prod_{r=0}^{n} p\left(\delta^{i}(t)\right) x\left(\delta^{n+1}(t)\right) \\
& \geq z(t)-\sum_{k=0}^{(n-1) / 2} \prod_{r=0}^{2 k} p\left(\delta^{i}(t)\right) z\left(\delta^{2 k+1}(t)\right),
\end{aligned}
$$

for $t \geq t_{2}$, where $t_{2} \geq t_{0}$ sufficiently large, and any odd positive integer $n$. Since $\delta^{2 k+1}(t) \leq \delta^{2 k}(t)$, we find

$$
z\left(\delta^{i}(t)\right) \leq z(t), \text { for } i=0,1, \ldots, n,
$$

which with (18) gives

$$
x(t) \geq\left(1-\sum_{k=0}^{(n-1) / 2} \prod_{r=0}^{2 k} p\left(\delta^{i}(t)\right)\right) z(t) .
$$

The proof is complete.

By replacing $\widehat{p}(t)$ instead of $p$ in the previous results, we can get the following corollary:

Corollary 2. Assume that (12) holds. Then every non-oscillatory solution of (1) tends to zero if $\int_{t_{0}}^{\infty} q(s) \mathrm{d} s=\infty$,

$$
\liminf _{t \rightarrow \infty} \int_{g(t)}^{t}(1-\hat{p}(g(s)))^{\alpha} \frac{q(s) g^{3 \alpha}(s)}{r(g(s))} \mathrm{d} s>\frac{6^{\alpha}}{\mu^{\alpha} \mathrm{e}}
$$

and

$$
\limsup _{t \rightarrow \infty} \int_{t_{0}}^{t}\left((1-\hat{p}(g(s)))^{\alpha} \theta^{\alpha}(s) q(s)\left(\frac{\mu}{2} g^{2}(s)\right)^{\alpha}-\left(\frac{\alpha}{\alpha+1}\right)^{\alpha+1} \frac{1}{r^{1 / \alpha}(s) \theta(s)}\right) \mathrm{d} s>0 .
$$




\section{New Oscillation Criteria}

For convenience, we denote:

$$
\begin{aligned}
& P_{k}(t):=\frac{1}{p\left(\delta^{-1}(t)\right)}\left(1-\frac{\left(\left(\delta^{-1}(t)\right)^{k-1} p\left(\delta^{-1}\left(\delta^{-1}(t)\right)\right)\right)^{-1}}{\left(\delta^{-1}\left(\delta^{-1}(t)\right)\right)^{1-k}}\right), \text { for } k=2, n, \\
& \Theta(s):=\frac{\alpha^{\alpha+1}}{(\alpha+1)^{\alpha+1}} \frac{r^{\alpha}\left(\delta^{-1}(g(s))\right)}{r^{1 / \alpha}(s) \theta(s)\left(\left(\delta^{-1}(g(s))\right)^{\prime}\right)^{\alpha}}, \theta(t)=\int_{t_{0}}^{\infty} r^{-1 / \alpha}(s) \mathrm{d} s
\end{aligned}
$$

and

$$
\widetilde{\Theta}(s)=\frac{\alpha^{\alpha+1}}{(\alpha+1)^{\alpha+1}} \frac{2^{\alpha} r^{\alpha}\left(\delta^{-1}(g(s))\right)}{r^{1 / \alpha}(s) \theta(s) \mu_{1}\left(\left(\delta^{-1}(g(s))\right)^{\prime}\left(\delta^{-1}(g(s))\right)^{2}\right)^{\alpha}} .
$$

Also, we define the Riccati substitutions

$$
\omega(t):=\frac{r(t)\left(z^{\prime \prime \prime}(t)\right)^{\alpha}}{\left(z^{\prime \prime}\left(\delta^{-1}(g(t))\right)\right)^{\alpha}}
$$

and

$$
\xi(t):=\frac{r(t)\left(z^{\prime \prime \prime}(t)\right)^{\alpha}}{z^{\alpha}\left(\delta^{-1}(g(t))\right)} .
$$

At studying the asymptotic behavior of positive solutions, there are three Cases $\left(S_{1}\right)-\left(S_{4}\right)$. We recall an existing criterion for Cases $\left(S_{1}\right)$ and $\left(S_{2}\right)$ in the following lemma:

Lemma 8 ([21]). Assume that $x$ be an eventually positive solution of (1). If (4) and (5) hold, then $z$ is neither satisfied $\left(S_{1}\right)$ nor $\left(S_{2}\right)$.

Lemma 9. Assume that $x$ be an eventually positive solution of (1) and

$$
\left(\delta^{-1}\left(\delta^{-1}(t)\right)\right)^{n-1}<\left(\delta^{-1}(t)\right)^{n-1} p\left(\delta^{-1}\left(\delta^{-1}(t)\right)\right) .
$$

Then

$$
x(t) \geq \frac{z\left(\delta^{-1}(t)\right)}{p\left(\delta^{-1}(t)\right)}-\frac{1}{p\left(\delta^{-1}(t)\right)} \frac{z\left(\delta^{-1}\left(\delta^{-1}(t)\right)\right)}{p\left(\delta^{-1}\left(\delta^{-1}(t)\right)\right)} .
$$

Proof. Assume that $x$ be an eventually positive solution of (1) on $\left[t_{0}, \infty\right)$. From the definition of $z(t)$, we see that

$$
p(t) x(\delta(t))=z(t)-x(t)
$$

and so

$$
p\left(\delta^{-1}(t)\right) x(t)=z\left(\delta^{-1}(t)\right)-z\left(\delta^{-1}(t)\right) .
$$

Repeating the same process, we obtain

$$
x(t)=\frac{1}{p\left(\delta^{-1}(t)\right)}\left(z\left(\delta^{-1}(t)\right)-\left(\frac{z\left(\delta^{-1}\left(\delta^{-1}(t)\right)\right)}{p\left(\delta^{-1}\left(\delta^{-1}(t)\right)\right)}-\frac{x\left(\delta^{-1}\left(\delta^{-1}(t)\right)\right)}{p\left(\delta^{-1}\left(\delta^{-1}(t)\right)\right)}\right)\right),
$$

which yields

$$
x(t) \geq \frac{z\left(\delta^{-1}(t)\right)}{p\left(\delta^{-1}(t)\right)}-\frac{1}{p\left(\delta^{-1}(t)\right)} \frac{z\left(\delta^{-1}\left(\delta^{-1}(t)\right)\right)}{p\left(\delta^{-1}\left(\delta^{-1}(t)\right)\right)} .
$$

Thus, (22) holds. This completes the proof. 
Lemma 10. Assume that $x$ is an eventually positive solution of (1) and

$$
\left(r(t)\left(z^{\prime \prime \prime}(t)\right)^{\alpha}\right)^{\prime} \leq-q(t) P_{1}^{\alpha}(g(t)) z^{\alpha}\left(\delta^{-1}(g(t))\right), \text { if } z \text { satisfies }\left(S_{3}\right)
$$

and

$$
\left(r(t)\left(z^{\prime \prime \prime}(t)\right)^{\alpha}\right)^{\prime}+q(t) P_{2}^{\alpha}(g(t)) z^{\alpha}\left(\delta^{-1}(g(t))\right) \leq 0, \text { if } z \text { satisfies }\left(S_{4}\right)
$$

Proof. Let case $\left(\mathbf{S}_{3}\right)$ holds. From Lemma 1, we have $z(t) \geq \frac{t}{2} z^{\prime}(t)$ and hence the function $t^{-1} z(t)$ is nonincreasing, which with the fact that $\delta(t) \leq t$ gives

$$
\left(\delta^{-1}(t)\right) z\left(\delta^{-1}\left(\delta^{-1}(t)\right)\right) \leq\left(\delta^{-1}\left(\delta^{-1}(t)\right)\right) z\left(\delta^{-1}(t)\right) .
$$

Combining (22) and (25), we see that

$$
\begin{aligned}
x(t) & \geq \frac{1}{p\left(\delta^{-1}(t)\right)}\left(1-\frac{\left(\delta^{-1}\left(\delta^{-1}(t)\right)\right)}{\delta^{-1}(t) p\left(\delta^{-1}\left(\delta^{-1}(t)\right)\right)}\right) z\left(\delta^{-1}(t)\right) \\
& =P_{2}(t) z\left(\delta^{-1}(t)\right) .
\end{aligned}
$$

From (1) and (26), we obtain

$$
\left(r(t)\left(z^{\prime \prime \prime}(t)\right)^{\alpha}\right)^{\prime} \leq-q(t) P_{n}^{\alpha}(g(t)) z^{\alpha}\left(\delta^{-1}(g(t))\right) .
$$

Thus, (23) holds. Assume that Case $\left(\mathbf{S}_{4}\right)$ holds. Since $\delta^{-1}(t) \leq \delta^{-1}\left(\delta^{-1}(t)\right)$. From (22), we see that

$$
\begin{aligned}
x(t) & \geq \frac{1}{p\left(\delta^{-1}(t)\right)}\left(1-\frac{1}{p\left(\delta^{-1}\left(\delta^{-1}(t)\right)\right)}\right) z\left(\delta^{-1}(t)\right) \\
& =P_{2}(t) z\left(\delta^{-1}(t)\right)
\end{aligned}
$$

which with (1) yields

$$
\left(r(t)\left(z^{\prime \prime \prime}(t)\right)^{\alpha}\right)^{\prime}+q(t) P_{2}^{\alpha}(g(t)) z^{\alpha}\left(\delta^{-1}(g(t))\right) \leq 0 .
$$

Thus, (24) holds. This completes the proof.

Lemma 11. Assume that $x$ be an eventually positive solution of $(1)$ and $\left(S_{3}\right)$ holds. If we have the function $\omega \in C^{1}[t, \infty)$ defined as (19), then

$$
\omega^{\prime}(t) \leq-q(t) P_{1}^{\alpha}(g(t))\left(\frac{\lambda}{2}\left(\delta^{-1}(g(t))\right)^{2}\right)^{\alpha}-\alpha \frac{\left(\delta^{-1}(g(t))\right)^{\prime}}{r^{1 / \alpha}(t) r\left(\delta^{-1}(g(t))\right)} \omega^{\frac{\alpha+1}{\alpha}}(t),
$$

for all $t>t_{1}$, where $t_{1}$ large enough.

Proof. Let $x$ is an eventually positive solution of (1). From Lemma (2), we get

$$
z\left(\delta^{-1}(g(t))\right) \geq \frac{\lambda}{2}\left(\delta^{-1}(g(t))\right)^{2} z^{\prime \prime}\left(\delta^{-1}(g(t))\right)
$$

for every $\lambda \in(0,1)$ and all sufficiently large $t$. Recalling that $r(t)\left(z^{\prime \prime \prime}(t)\right)^{\alpha}$ is decreasing, we get

$$
r\left(\delta^{-1}(g(t))\right)\left(z^{\prime \prime \prime}\left(\delta^{-1}(g(t))\right)\right)^{\alpha} \geq r(t)\left(z^{\prime \prime \prime}(t)\right)^{\alpha} .
$$


This yields

$$
\left(z^{\prime \prime \prime}\left(\delta^{-1}(g(t))\right)\right)^{\alpha} \geq \frac{r(t)}{r\left(\delta^{-1}(g(t))\right)}\left(z^{\prime \prime \prime}(t)\right)^{\alpha} .
$$

From the definition of $\omega(t)$, we see that $\omega(t)<0$ for $t \geq t_{1}$. By differentiating, we find

$$
\omega^{\prime}(t)=\frac{\left(r(t)\left(z^{\prime \prime \prime}(t)\right)^{\alpha}\right)^{\prime}}{\left(z^{\prime \prime}\left(\delta^{-1}(g(t))\right)\right)^{\alpha}}-\alpha \frac{r(t)\left(z^{\prime \prime \prime}(t)\right)^{\alpha} z^{\prime \prime \prime}\left(\delta^{-1}(g(t))\right)\left(\delta^{-1}(g(t))\right)^{\prime}}{\left(z^{\prime \prime}\left(\delta^{-1}(g(t))\right)\right)^{\alpha+1}} .
$$

From (19), (30) and (31), we get

$$
\begin{aligned}
\omega^{\prime}(t) & \leq-q(t) P_{1}^{\alpha}(g(t)) \frac{z^{\alpha}\left(\delta^{-1}(g(t))\right)}{\left(z^{\prime \prime}\left(\delta^{-1}(g(t))\right)\right)^{\alpha}}-\alpha \frac{r(t)\left(\delta^{-1}(g(t))\right)^{\prime}}{r\left(\delta^{-1}(g(t))\right)} \frac{\left(z^{\prime \prime \prime}(t)\right)^{\alpha+1}}{\left(z^{\prime \prime}\left(\delta^{-1}(g(t))\right)\right)^{\alpha+1}} \\
& \leq-q(t) P_{1}^{\alpha}(g(t))\left(\frac{\lambda}{2}\left(\delta^{-1}(g(t))\right)^{2}\right)^{\alpha}-\alpha \frac{\left(\delta^{-1}(g(t))\right)^{\prime}}{r^{1 / \alpha}(t) r\left(\delta^{-1}(g(t))\right)} \omega^{\frac{\alpha+1}{\alpha}}(t) .
\end{aligned}
$$

The proof is complete.

Lemma 12. Assume that $x$ be an eventually positive solution of (1) and $\left(S_{4}\right)$ holds. If we have the function $\zeta \in C^{1}[t, \infty)$ defined as (20), then

$$
\zeta^{\prime}(t) \leq-q(t) P_{2}^{\alpha}(g(t))-\alpha \frac{\mu_{1}\left(\delta^{-1}(g(t))\right)^{\prime}\left(\delta^{-1}(g(t))\right)^{2}}{2 r^{1 / \alpha}\left(\delta^{-1}(g(t))\right)} \xi^{\alpha+1}(t)
$$

for all $t>t_{1}$, where $t_{1}$ large enough.

Proof. Let $x$ is an eventually positive solution of (1). From the definition of $\xi(t)$, we see that $\xi(t)<$ 0 for $t \geq t_{1}$. By differentiating, we find

$$
\xi^{\prime}(t) \leq-q(t) P_{2}^{\alpha}(g(t))-\alpha \frac{r(t)\left(z^{\prime \prime \prime}(t)\right)^{\alpha}\left(\delta^{-1}(g(t))\right)^{\prime} z^{\prime}\left(\delta^{-1}(g(t))\right)}{z^{\alpha+1}\left(\delta^{-1}(g(t))\right)} .
$$

From Lemma 2 and (31), we get

$$
z^{\prime}\left(\delta^{-1}(g(t))\right) \geq \frac{\mu_{1}}{2}\left(\delta^{-1}(g(t))\right)^{2}\left(\frac{r(t)}{r\left(\delta^{-1}(g(t))\right)}\right)^{1 / \alpha} z^{\prime \prime \prime}(t)
$$

for all $\mu_{1} \in(0,1)$ and every sufficiently large $t$. Thus, by (20), (33) and (34), we get

$$
\xi^{\prime}(t) \leq-q(t) P_{2}^{\alpha}(g(t))-\alpha \frac{\mu_{1}\left(\delta^{-1}(g(t))\right)^{\prime}\left(\delta^{-1}(g(t))\right)^{2}}{2 r^{1 / \alpha}\left(\delta^{-1}(g(t))\right)} \xi^{\alpha+1}(t) .
$$

The proof is complete.

Theorem 2. Assume that (4) and (5) hold. If

$$
\int_{t_{0}}^{\infty}\left(q(s) P_{1}^{\alpha}(g(s))\left(\frac{\lambda}{2}\left(\delta^{-1}(g(t))\right)^{2}\right)^{\alpha} \theta^{\alpha}(s) d s-\Theta(s)\right) \mathrm{d} s=\infty
$$

and

$$
\int_{t_{0}}^{\infty}\left(q(s) P_{2}^{\alpha}(g(s)) \theta^{\alpha}(s) d s-\widetilde{\Theta}(s)\right) \mathrm{d} s=\infty,
$$

for some $\mu, \lambda \in(0,1)$, then every solution of $(1)$ is oscillatory. 
Proof. Assume the contrary that $x$ is a positive solution of (1). From Lemma 4, we have cases $\left(S_{1}\right)-\left(S_{4}\right)$. From Lemmas $8, z$ is neither satisfied $\left(S_{1}\right)$ nor $\left(S_{2}\right)$. Suppose that case $\left(\mathbf{S}_{3}\right)$ holds. From Lemma 11, we get that (29) holds. Multiplying this inequality by $\theta^{\alpha}(t)$ and integrating the resulting inequality from $t_{1}$ to $t$, we get

$$
\begin{aligned}
\theta^{\alpha}(t) \omega(t)-\theta^{\alpha}\left(t_{1}\right) \omega\left(t_{1}\right)+\alpha \int_{t_{1}}^{t} r^{\frac{-1}{\alpha}}(s) \theta^{\alpha-1}(s) \omega(s) d s \\
\leq-\int_{t_{1}}^{t} q(s) P_{1}^{\alpha}(g(s))\left(\frac{\lambda}{2}\left(\delta^{-1}(g(t))\right)^{2}\right)^{\alpha} \theta^{\alpha}(s) d s \\
\quad-\alpha \int_{t_{1}}^{t} \frac{\theta^{\alpha}(s)\left(\delta^{-1}(g(s))\right)^{\prime} g^{\prime}(s)}{r^{1 / \alpha}(s) r\left(\delta^{-1}(g(s))\right)} \omega^{\frac{\alpha+1}{\alpha}}(s) d s .
\end{aligned}
$$

We set

$$
D:=r^{\frac{-1}{\alpha}}(s) \theta^{\alpha-1}(s), C:=\frac{\theta^{\alpha}(s)\left(\delta^{-1}(g(s))\right)^{\prime} g^{\prime}(s)}{r^{1 / \alpha}(s) r\left(\delta^{-1}(g(s))\right)}, y:=-\omega(s) .
$$

Using Lemma 12, we find

$$
\begin{aligned}
& r^{\frac{-1}{\alpha}}(s) \theta^{\alpha-1}(s) \omega(s)-\frac{\theta^{\alpha}(s)\left(\delta^{-1}(g(s))\right)^{\prime} g^{\prime}(s)}{r^{1 / \alpha}(s) r\left(\delta^{-1}(g(s))\right)} \omega^{\frac{\alpha+1}{\alpha}} \\
\leq & \frac{\alpha^{\alpha+1}}{(\alpha+1)^{\alpha+1}} \frac{r^{\alpha}\left(\delta^{-1}(g(s))\right)}{r^{1 / \alpha}(s) \theta(s)\left(\left(\delta^{-1}(g(s))\right)^{\prime}\right)^{\alpha}} .
\end{aligned}
$$

From (37), for every $\lambda \in(0,1)$, and all sufficiently large $t$, we obtain

$$
\int_{t_{1}}^{t}\left(q(s) P_{1}^{\alpha}(g(s))\left(\frac{\lambda}{2}\left(\delta^{-1}(g(t))\right)^{2}\right)^{\alpha} \theta^{\alpha}(s) d s-\Theta(s)\right) d s \leq \theta^{\alpha}\left(t_{1}\right) g\left(t_{1}\right)+1,
$$

but this contradicts (35). The proof is complete. Let case $\left(\mathbf{S}_{3}\right)$ holds. Using Lemma 12, we have that (32) holds. Multiplying this inequality by $\theta^{\alpha}(t)$ and integrating the resulting inequality from $t_{1}$ to $t$, we get

$$
\begin{aligned}
& \theta^{\alpha}(t) \xi(t)-\theta^{\alpha}\left(t_{1}\right) \xi\left(t_{1}\right)+\alpha \int_{t_{1}}^{t} r^{\frac{-1}{\alpha}}(s) \theta^{\alpha-1}(s) \xi(s) d s \\
\leq & -\int_{t_{1}}^{t} q(s) P_{2}^{\alpha}(g(s)) \theta^{\alpha}(s) d s-\alpha \int_{t_{1}}^{t} \frac{\mu_{1} \theta^{\alpha}(s)\left(\delta^{-1}(g(s))\right)^{\prime} g^{\prime}(s)\left(\delta^{-1}(g(s))\right)^{2}}{2 r^{1 / \alpha}(s) r\left(\delta^{-1}(g(s))\right)} \xi^{\frac{\alpha+1}{\alpha}}(s) d s .
\end{aligned}
$$

We set

$$
D:=r^{\frac{-1}{\alpha}}(s) \theta^{\alpha-1}(s), C:=\frac{\mu_{1} \theta^{\alpha}(s)\left(\delta^{-1}(g(s))\right)^{\prime} g^{\prime}(s)\left(\delta^{-1}(g(s))\right)^{2}}{2 r^{1 / \alpha}(s) r\left(\delta^{-1}(g(s))\right)}, y:=-\xi(s)
$$

Applying Lemma 3, for every $\mu_{1} \in(0,1)$, we obtain

$$
\begin{aligned}
& r^{\frac{-1}{\alpha}}(s) \theta^{\alpha-1}(s) \xi(s)-\frac{\mu_{1} \theta^{\alpha}(s)\left(\delta^{-1}(g(s))\right)^{\prime} g^{\prime}(s)\left(\delta^{-1}(g(s))\right)^{2}}{2 r^{1 / \alpha}(s) r\left(\delta^{-1}(g(s))\right)} \xi^{\frac{\alpha+1}{\alpha}} \\
\leq & \frac{\alpha^{\alpha+1}}{(\alpha+1)^{\alpha+1}} \frac{2^{\alpha} r^{\alpha}\left(\delta^{-1}(g(s))\right)}{r^{1 / \alpha}(s) \theta(s) \mu_{1}\left(\left(\delta^{-1}(g(s))\right)^{\prime}\left(\delta^{-1}(g(s))\right)^{2}\right)^{\alpha}}
\end{aligned}
$$


which implise that

$$
\int_{t_{1}}^{t}\left(q(s) P_{2}^{\alpha}(g(s)) \theta^{\alpha}(s) d s-\widetilde{\Theta}(s)\right) d s \leq \theta^{\alpha}\left(t_{1}\right) g\left(t_{1}\right)+1,
$$

but this contradicts (36). The proof is complete.

Example 1. Consider the equation

$$
\left(t^{2}\left(x(t)+16 x\left(\frac{t}{2}\right)\right)^{\prime \prime \prime}\right)^{\prime}+q_{0} x\left(\frac{t}{2}\right)=0,
$$

where $t \geq 1, q_{0}>0$. We note that $r(t)=t^{2}, p(t)=16, \delta(t)=g(t)=1 / 2 t$ and $q(t)=q_{0}$. Moreover, we get

$$
P_{1}(t)=\frac{7}{128}, P_{2}(t)=\frac{1}{32}, \theta(t)=\frac{1}{t}, \Theta(t)=\frac{t}{4}
$$

and

$$
\widetilde{\Theta}(t)=\frac{1}{2 t} .
$$

Thus, we find

$$
\begin{aligned}
& \int_{t_{0}}^{\infty}\left(q(s) P_{1}^{\alpha}(g(s))\left(\frac{\lambda}{2}\left(\delta^{-1}(g(t))\right)^{2}\right)^{\alpha} \theta^{\alpha}(s) d s-\Theta(s)\right) \mathrm{d} s \\
= & \left(\frac{7 q_{0}}{256}-\frac{1}{4}\right) \int_{t_{0}}^{\infty} s \mathrm{~d} s \\
= & \infty \text { if } q_{0}>9.14
\end{aligned}
$$

and

$$
\begin{aligned}
& \int_{t_{0}}^{\infty}\left(q(s) P_{2}^{\alpha}(g(s)) \theta^{\alpha}(s) d s-\widetilde{\Theta}(s)\right) \mathrm{d} s \\
= & \left(\frac{q_{0}}{32}-\frac{1}{2}\right) \int_{t_{0}}^{\infty} \frac{1}{s} \mathrm{~d} s \\
= & \infty \text { if } q_{0}>16 .
\end{aligned}
$$

Therefore, applying Theorem 2, we have that every solution of (38) is oscillatory if $q_{0}>16$.

Example 2. Consider the equation

$$
\left(t^{2}\left(x(t)+4 x\left(\frac{t}{2}\right)\right)^{\prime \prime \prime}\right)^{\prime}+q_{0} x\left(\frac{t}{2}\right)=0,
$$

where $t \geq 1, q_{0}>0$. We note that $r(t)=t^{2}, p(t)=4, \delta(t)=g(t)=1 / 2 t$ and $q(t)=q_{0}$. Thus, it's easy to see that (4) and (5) are satisfied. Moreover, we have

$$
P_{1}(t)=\frac{1}{8}, P_{2}(t)=\frac{3}{16} .
$$

Hence, Condition (35) and (36) become

$$
q_{0}>4
$$

and

$$
q_{0}>\frac{8}{3}
$$


respectively. It's easy to see that (40) implies (41). Therefore, by Theorem 2, we conclude that (39) is oscillatory if (40) holds.

Remark 1. The results of this paper can be extended to the more general equation of the form

$$
\left(r(t)\left(z^{(n-1)}(t)\right)^{\alpha}\right)^{\prime}+q(t) x^{\beta}(g(t))=0 .
$$

The statement and the formulation of the results are left to the interested reader.

Remark 2. One can easily see that the results obtained in [25] cannot be applied to Theorem 2, so our results are new.

\section{Conclusions}

This paper is concerned with oscillatory behavior of a class of fourth-order delay differential equations. Using a Riccati transformation, a new asymptotic criterion for (1) is presented. In future work, we will aim to present a new comparison theorem that compares the higher-order Equation (1) with first-order equations. There are numerous results concerning the oscillation criteria of first order equations, which include various forms of criteria such as Hille/Nehari, Philos, etc. This allows us to obtain various criteria for the oscillation of (1). Further, we can try to get some oscillation criteria of (1) if $z(t):=x(t)-p(t) x(\delta(t))$.

Author Contributions: The authors claim to have contributed equally and significantly in this paper. All authors have read and agreed to the published version of the manuscript.

Funding: This research received no external funding.

Acknowledgments: The authors thank the reviewers for for their useful comments, which led to the improvement of the content of the paper.

Conflicts of Interest: The authors declare no conflict of interest.

\section{References}

1. Agarwal, R.P.; Shieh, S.L.; Yeh, C.C. Oscillation criteria for second order retarded differential equations. Math. Comput. Model. 1997, 26, 1-11. [CrossRef]

2. Bohner, M.; Grace, S.R.; Jadlovska, I. Oscillation criteria for second-order neutral delay differential equations. Electron. J. Qual. Theory Differ. Equ. 2017, 2017, 60. [CrossRef]

3. Kitamura, Y.; Kusano, T. Oscillation of first-order nonlinear differential equations with deviating arguments, Proc. Amer. Math. Soc. 1980, 78, 64-68. [CrossRef]

4. Philos, C.G. On the existence of nonoscillatory solutions tending to zero at $\infty$ for differential equations with positive delays. Arch. Math. 1981, 36, 168-178. [CrossRef]

5. Agarwal, R.P.; Bohner, M.; Li, T.; Zhang, C. A new approach in the study of oscillatory behavior of even-order neutral delay diferential equations, Appl. Math. Comput. 2013, 225, 787-794.

6. Agarwal, R.; Grace, S.; O'Regan, D. Oscillation Theory for Difference and Functional Differential Equations; Kluwer Acad. Publ.: Dordrecht, The Netherlands, 2000.

7. Baculikova, B.; Dzurina, J. Oscillation theorems for second-order nonlinear neutral differential equations. Comput. Math. Appl. 2011, 62, 4472-4478. [CrossRef]

8. Bazighifan, O.; Cesarano, C. Some New Oscillation Criteria for Second-Order Neutral Differential Equations with Delayed Arguments. Mathematics 2019, 7, 619. [CrossRef]

9. Bazighifan, O.; Elabbasy, E.M.; Moaaz, O. Oscillation of higher-order differential equations with distributed delay. J. Inequal. Appl. 2019, 55, 1-9. [CrossRef]

10. Chatzarakis, G.E.; Elabbasy, E.M.; Bazighifan, O. An oscillation criterion in 4th-order neutral differential equations with a continuously distributed delay. Adv. Differ. Equ. 2019, 336, 1-9.

11. Chatzarakis, G.E.; Li, T. Oscillations of differential equations generated by several deviating arguments. Adv. Differ. Equ. 2017, 2017, 1-24. [CrossRef] 
12. Chatzarakis, G.E.; Li, T. Oscillation criteria for delay and advanced differential equations with nonmonotone arguments. Complexity 2018, 2018, 1-18. [CrossRef]

13. El-Nabulsi, R.A.; Moaaz, O.; Bazighifan, O. New Results for Oscillatory Behavior of Fourth-Order Differential Equations. Symmetry 2020, 12, 136. [CrossRef]

14. Elabbasy, E.M.; Cesarano, C.; Bazighifan, O.; Moaaz, O. Asymptotic and oscillatory behavior of solutions of a class of higher order differential equation. Symmetry 2019, 11, 1434. [CrossRef]

15. Bazighifan, O.; Cesarano, C. A Philos-Type Oscillation Criteria for Fourth-Order Neutral Differential Equations. Symmetry 2020, 12, 379. [CrossRef]

16. Hale, J.K. Theory of Functional Differential Equations; Springer: New York, NY, USA, 1977.

17. Li, T.; Han, Z.; Zhao, P.; Sun, S. Oscillation of even-order neutral delay differential equations. Adv. Differ. Equ. 2010, 2010, 1-9. [CrossRef]

18. Kiguradze, I.T.; Chanturiya, T.A. Asymptotic Properties of Solutions of Nonautonomous Ordinary Differential Equations; Kluwer Acad. Publ.: Dordrecht, The Netherlands, 1993.

19. Moaaz, O. New criteria for oscillation of nonlinear neutral differential equations. Adv. Differ. Eqs. 2019, $2019,484$. [CrossRef]

20. Moaaz, O.; Elabbasy, E.M.; Bazighifan, O. On the asymptotic behavior of fourth-order functional differential equations. Adv. Differ. Equ. 2017, 2017, 261. [CrossRef]

21. Moaaz, O.; Awrejcewicz, J.; Bazighifan, O. A New Approach in the Study of Oscillation Criteria of Even-Order Neutral Differential Equations. Mathematics 2020, 2020, 179. [CrossRef]

22. Moaaz, O.; El-Nabulsi, R.; Bazighifan, O. Oscillatory behavior of fourth-order differential equations with neutral delay. Symmetry 2020, 12, 371. [CrossRef]

23. Moaaz, O.; Elabbasy, E.M.; Muhib, A. Oscillation criteria for even-order neutral differential equations with distributed deviating arguments. Adv. Differ. Equ. 2019, 297,1-10. [CrossRef]

24. Moaaz, O.; Elabbasy, E.M.; Shaaban, E. Oscillation criteria for a class of third order damped differential equations. Arab. J. Math. Sci. 2018, 24, 16-30. [CrossRef]

25. Tang, S.; Li, T.; Thandapani, E. Oscillation of higher-order half-linear neutral differential equations. Demonstr. Math. 2013, 1, 101-109. [CrossRef]

26. Xing, G.; Li, T.; Zhang, C. Oscillation of higher-order quasi linear neutral differential equations. Adv. Differ. Equ. 2011, 2011, 1-10. [CrossRef]

27. Zafer, A. Oscillation criteria for even order neutral differential equations. Appl. Math. Lett. 1998, 11, 21-25. [CrossRef]

28. Zhang, Q.; Yan, J. Oscillation behavior of even order neutral differential equations with variable coefficients. Appl. Math. Lett. 2006, 19, 1202-1206. [CrossRef] 